\title{
Incidencia en dispensación de estupefaciente y psicótropo. ¿La paciente oncológica se queda sin analgesia?
}

\author{
Nerea Acuña Elvira \\ Licenciada en Farmacia. Doctora en Ciencias de la Salud. Farmacéutica comunitaria en Lanzarote.
}

\section{PALABRAS CLAVE}

Buenas prácticas de dispensación, estupefaciente, psicótropo, servicios comunitarios de farmacia

\section{KEYWORDS}

Good dispensing practices, narcotic, psychotropic, pharmaceutical services

\begin{abstract}
RESUMEN
Paciente de 36 años con antecedentes de cáncer que tiene prescrito fentanilo 0,2 mg y Dormicum ${ }^{\circledR}$ 7,5 mg. Acude a nuestra farmacia un sábado a las 11:00 para retirar ambos medicamentos porque no tiene suficiente para el fin de semana. Al entrar en su plan de tratamiento, comprobamos que dicho plan está anulado y que la totalidad de los medicamentos aparecen como "no dispensables". Al tratarse de medicamentos que necesitan prescripción derivamos al centro de salud con servicio de urgencias que está abierto los sábados, solicitando al médico de urgencias que actualice el plan de tratamiento, ya que hemos comprobado que la paciente no tiene medicación suficiente para el fin de semana y ella nos indica que ningún médico le ha suspendido el tratamiento. La paciente acude al centro médico, y tras 1,5 horas nos llama llorando diciéndonos que la facultativo que la atendió no le actualiza el plan de tratamiento. La derivamos al hospital y, como no queda tiempo suficiente para que llegue a recoger la medicación porque cerramos a las 14:00, llamamos a las farmacias de guardia para informarnos de si disponen de la medicación, no la tienen y no tendrían forma de conseguirla hasta el lunes. Damos el teléfono personal a la paciente para que nos informe de la situación, nos llama al salir del hospital, ya son las 16:00 y nuestra farmacia está cerrada. El médico del hospital nos informa de que la conducta de la médica del centro de salud de urgencias es totalmente inapropiada, que la paciente bajo ningún concepto puede suspender la medicación de forma gradual. Decidimos desplazarnos a la farmacia y dispensarle la medicación.
\end{abstract}

Incidence in the dispensing of narcotic and psychotropic drugs. Does de cancer patient run out of analgesia?

\section{ABSTRACT}

36-year-old patient with a history of cancer prescribed Fentanyl $0.2 \mathrm{mg}$ and Dormicum ${ }^{\circledR} 7.5 \mathrm{mg}$. She goes to our pharmacy office on a Saturday at 11:00 to pick up both medications because she doesn't have enough for the weekend. When we enter your treatment plan, we verify that this plan is canceled and that all the medications appear as "not dispensable". When dealing with medications that need a prescription, we refer to the health center with an emergency service that is open on Saturdays, asking the emergency doctor to update the treatment plan since we have verified that the patient does not have enough medication for the weekend and she tells us that no doctor has stopped your treatment. The patient goes to the medical center, after 1.5 hours she calls us crying telling us that the doctor who treated her does not update her treatment plan. We refer her to the hospital and since there is not enough time for her to pick up the medication because we close at 2:00 p.m. we call the pharmacies on duty to inform us if they have the medication, they do not have it and they would not have a way to get it until Monday. We give the patient the personal telephone number so that she can inform us of the situation, she calls us when she leaves the hospital, it is already 4:00 p.m., our pharmacy is closed. The hospital doctor informs us that the conduct of the emergency health center doctor is totally inappropriate, that the patient can under no circumstances gradually discontinue the medication. We decided to go to the pharmacy and dispense the medication.

Financiación: ninguna.

Conflicto de intereses: ninguno.

Cite este artículo como: Acuña N. Incidencia en dispensación de estupefaciente y psicótropo. ¿La paciente oncológica se queda sin analgesia?. Farmacéuticos Comunitarios. 2021 Apr 19; 13 (2): 49-52. doi:10.33620/FC.2173-9218.(2021/ Vol13).002.08

Correspondencia: Nerea Acuña Elvira (nereacue@hotmail.com).

ISSN 1885-8619 @SEFAC (Sociedad Española de Farmacia Clínica, Familiar y Comunitaria). Todos los derechos reservados.
Aceptado: 29/3/2021

Disponible online: 19/4/2021 


\section{Presentación}

Paciente de 36 años con antecedentes de cáncer que acude a nuestra farmacia a recoger la medicación desde hace varios años. Es por ello que conocemos muy bien su medicación y su evolución terapéutica. Ha estado largas temporadas ingresada en el hospital por, entre otros motivos, no controlar el dolor. Actualmente ha mejorado mucho, ha conseguido llegar a normopeso y su estado aparente es bueno. Acude a la farmacia acompañada por sus dos hijas un sábado a las 11:00 de la mañana para retirar fentanilo y Dormicum ${ }^{\circledR}$. Al entrar en su plan de tratamiento electrónico utilizando su tarjeta sanitaria, comprobamos que todos sus medicamentos aparecen como "no dispensables, pendientes de revisión”. La paciente empieza a ponerse muy nerviosa porque no tiene medicación para el fin de semana, y nuestra farmacia, que según ella es la única que tiene el estupefaciente, cierra a las 14:00. En la tabla 1 se presenta el tratamiento que estaba recibiendo la paciente.

\section{Evaluación}

Entrando en el histórico de dispensación y estudiando las posologías $(1,2,3)$, comprobamos que efectivamente la paciente no tiene medicación suficiente para cubrir el fin de semana. Estudiamos las fichas técnicas de los medicamentos (4) y comprobamos que la retirada radical del fentanilo y del psicótropo pueden causar síndrome de abstinencia y deben hacerse bajo un estrecho seguimiento médico.

Evidentemente, y aunque conozcamos perfectamente a la paciente y su situación terapéutica, no podemos dispensarle los medicamentos (2) que nos solicita sin la correspondiente prescripción médica, por lo que derivamos al centro de salud con servicio de urgencias correspondiente para que le actualicen el plan de tratamiento, suponiendo que antes de nuestro cierre la situación quedará resuelta.

\section{Intervención}

Pasada una hora y media, la paciente contacta con nosotros vía telefónica y nos informa de que no le han actualizado el plan de tratamiento, y le pide a la farmacéutica que, por favor, le adelante la medicación que precisa, porque ha llamado a las farmacias de guardia y ninguna dispone del estupefaciente ni pueden recibir pedidos hasta el lunes en caso de que consiguiera que le actualizaran el plan de tratamiento en el hospital.
Ante esta situación decidimos contactar con la médica de urgencias en el centro de salud con la que acababa de hablar la paciente, nos informa de que "lo que ha pasado con el plan de tratamiento es que se ha caducado y que para renovarlo la paciente tendría que haber acudido a su médico de atención primaria de lunes a viernes, que eso era un servicio de urgencias en el que no se realizan esas gestiones". Le insistimos en que se trata de un caso muy particular con tratamiento estupefaciente y con psicótropos que no pueden suspenderse de forma radical y que los planes de tratamiento, debido a la situación actual de pandemia de COVID-19, se actualizan automáticamente en la tarjeta sanitaria de los pacientes sin que ellos puedan ver ni sean informados de fechas de caducidad. Es más, al actualizarse automáticamente los tratamientos crónicos, no pueden darse estas situaciones de planes de tratamiento caducados, pero ella insiste en que no puede hacer nada.

La paciente está muy nerviosa y llorando. La tranquilizamos, le damos el teléfono personal de la farmacéutica y le decimos que vamos a llamar a todas las farmacias de guardia de la isla a ver si alguna dispone de su tratamiento. Derivamos al hospital para que intente que le actualicen el plan de tratamiento.

Tabla 1 Tratamiento crónico

\begin{tabular}{|l|l|}
\hline Prescripción & Instrucciones al paciente \\
\hline Pantoprazol $20 \mathrm{mg} 56$ comprimidos oral & 1 con el desayuno \\
\hline Ondansetron $8 \mathrm{mg} 15$ comprimidos oral & 1 cada 12 horas \\
\hline Lactulosa $10 \mathrm{~g} / \mathrm{sob} 50$ sobres & 1 cada 24 horas \\
\hline Colecalciferol 25000 Ul 4 capsulas & 1 cada 2 semanas \\
\hline Fentanilo $0,2 \mathrm{mg} 30$ comprimidos & 3 comprimidos cada 4 horas. Sublingual \\
\hline Pregabalina $75 \mathrm{mg} 56$ capsulas & 1 cada 24 horas \\
\hline Midazolam $7,5 \mathrm{mg} 20$ comprimidos & 1 cada 12 horas \\
\hline Mirtazapina $30 \mathrm{mg} 30$ comprimidos & 1 con la cena \\
\hline Al0 dia anatómico pants talla pequeña & 1 cada 8 horas \\
\hline $\begin{array}{l}\text { Apósitos B ostom aro res sint mic conform } 2 \text { placa plana bord aro } 55 \mathrm{~mm} 13-40 \\
\text { mm } 5 \mathrm{U}\end{array}$ & 1 cada 8 horas \\
\hline $\begin{array}{l}\text { Bolsas colost sist doble plac conform } 2 \text { cerrada plana midi opaca aro } 55 \mathrm{~mm} \\
13-40 \text { mm } 30+7\end{array}$ & \begin{tabular}{l}
1 cada 6 horas \\
\hline Suportan drink 24 botellas $200 \mathrm{ml}$ sabor capuchino
\end{tabular} \\
\hline
\end{tabular}




\section{Resultado}

Llamamos a las farmacias de guardia y, efectivamente, ninguna dispone de los medicamentos que la paciente necesita para el fin de semana. Contactamos con ella y le informamos de que ninguna farmacia de la isla dispone de la medicación, pero que no se preocupe, que nosotros se la dispensaremos si le actualizan el plan de tratamiento, aunque ya estemos cerrados.

A las 16:00 la farmacéutica recibe la llamada de la paciente, nos pasa al médico del hospital que nos informa de que la conducta de la médica de urgencias ha sido totalmente inapropiada, que tomarán medidas y que, por favor, le dispensemos la medicación, por tratarse de un tratamiento que no es posible suspender radicalmente, ya que acabaría ocasionando nuevamente el ingreso hospitalario de la paciente.

Acudimos a la farmacia y dispensamos el estupefaciente y el psicótropo.

\section{Discusión}

Se trata de la primera vez que nos enfrentamos a la situación de que un médico de urgencias se niega a actualizar el plan de tratamiento de una paciente. La situación añadida de tratarse de medicamentos estupefacientes y psicótropos, ser sábado y encontrarnos en una isla en la que sólo hay un almacén de cooperativa farmacéutica en la que no se dispone de todos los medicamentos suma aún más dificultad a este caso.

Intentamos contactar con el servicio jurídico de nuestro colegio (COF), pero fue imposible al tratarse de sábado, así que, tras estudiar todas las opciones, decidimos resolver la situación de la forma que nos pareció más deontológicamente correcta.

¿Pueden los pacientes sufrir las consecuencias de los errores administrativos o tecnológicos que puedan sucederse en las actualizaciones automáticas de los planes de tratamiento? ¿Qué hubiese sucedido de haber sido otra nuestra actuación y haber decidido no dispensar la medicación hasta el lunes?

Quizás en épocas de pandemia como la que estamos viviendo, en la que muchísimas gestiones se hacen de forma inmediata y telemática, debería implantarse un sistema de comunicación directa farmacéutico-médico que tuviera disponibilidad continua y que ahorrase a los pacientes los engorrosos trámites, esperas y perjuicios en sus tratamientos terapéuticos. Se vuelve a poner de manifiesto (5) la importancia de compartir la historia clínica de los pacientes entre los diferentes profesionales de la salud.

\section{Referencias bibliográficas}

1. Acuña Elvira N. Consulta farmacéutica: intoxicación por gemfibrozilo. Farmacéuticos Comunitarios. 2017 Jun 30;9(2):49-51. doi:10.5672/FC.21739218.(2017/Vol9).002.06

2. Acuña Elvira N. Día a día en la oficina de farmacia. Manual Práctico. ISBN 978-620-0-36853-9.

3. Acuña Elvira N. Consulta farmacéutica: intoxicaciones medicamentosas. ISBN 978-620-2-09965-3.

4. Consejo General de Colegios Oficiales de Farmacéuticos. Bot Plus Web (Base de datos en internet) 2020. (Consultada 14/11/2020) Disponible en: https:// botplusweb.portalfarma.com

5. García-Delgado Morente A. Importancia de compartir la historia clínica entre los miembros del equipo de salud. Farmacéuticos Comunitarios. 2014; 6 (Supl. 1). Disponible en: https:// www.farmaceuticoscomunitarios.org/ es/journal-article/importancia-compartir-historia-clinica-entre-miembros-del-equipo-salud 


\section{Nota de la Dirección}

Ante el interés humano y legal que presenta el caso expuesto hemos consultado con un asesor jurídico las implicaciones legales en caso de que la farmacia hubiera dispensado el estupefaciente antes de disponer de la receta. El comentario se expone a continuación.

\section{Comentario bioético y legal}

\section{Fernando Abellán-García Sánchez \\ Doctor en Medicina Legal y Forense por la Universidad Complutense de Madrid.}

El caso clínico plantea un problema de "dispensación excepcional” en el que el farmacéutico se ve ante el dilema ético de facilitar la medicación para asegurar la continuidad del tratamiento o cumplir estrictamente la normativa farmacéutica que condiciona la dispensación a la presentación de la receta, con la particularidad de que cuando se trata de estupefacientes y psicótropos aquélla es todavía más exigente que en el resto de medicamentos de prescripción.

Doctrinalmente, en los supuestos de dispensación excepcional han de darse dos condiciones de manera acumulativa: la primera de ellas, que tenga que dispensarse un medicamento que haya sido prescrito previamente de alguna manera -verbalmente, fax, informe de urgencia, etc.- aunque no se disponga de la receta; y la segunda condición, que exista una situación de urgencia o necesidad que justifique la dispensación.

Si se proyecta lo anterior en el caso analizado, puede afirmarse que consta la prescripción y su vigencia a través de la conversación mantenida entre la farmacéutica y la médica del hospital, quien telefónicamente solicita "que, por favor, le dispensemos la medicación ya que se trata de un tratamiento que no es posible suspender radicalmente". Es muy conveniente anotar el nombre del médico para poder contactar con él posteriormente si fuera necesario. Asimismo, todo indica que lo sucedido a la paciente obedece a un error administrativo o informático. También es importante que en la farmacia se conoce "muy bien su medicación y su evolución terapéutica".

Igualmente se cumple la condición relativa a la situación de urgencia o necesidad, por cuanto la paciente no tiene medicación para cubrir el fin de semana y la médica se pronuncia en el sentido de que "bajo ningún concepto puede suspender la medicación de forma gradual”, añadiendo que si se hiciera "acabaría ocasionando nuevamente el ingreso hospitalario de la paciente”. Es decir, perjudicaría gravemente su salud. La propia farmacéutica comprueba leyendo las fichas técnicas que la retirada radical del fentanilo y del psicótropo pueden causar síndrome de abstinencia. Finalmente, la paciente "está muy nerviosa y llorando", lo que pone de manifiesto su situación de angustia.

Teniendo en cuenta el conjunto de circunstancias presentes, el farmacéutico debe resolver su dilema ético en un sentido u otro; esto es, dispensando la medicación o absteniéndose de hacerlo, a sabiendas de que cualquiera de las dos opciones lesionará otro valor importante: si no dispensa se perjudicará la salud de la paciente y si sí lo hace contravendrá la norma al no contar con la receta. En este estado de cosas, se antoja correcta la decisión adoptada, que inicialmente se decanta por el curso de acción intermedio de derivar a la paciente al centro de salud para que consiga la receta, y, posteriormente, a la vista de que no es posible, decide proceder a la dispensación entendiendo que lo más valioso es preservar la salud de la paciente.

A la misma conclusión se llegaría en el plano estrictamente legal, pues la farmacéutica, en caso de ser acusada de cometer la infracción de dispensar sin receta, se podría acoger para defender su actuación a lo que se denomina el "estado de necesidad justificante", que opera como factor de exoneración de la culpabilidad cuando se puede probar debidamente. Se trata de una construcción del derecho penal (art. 20.5 del Código Penal) que se proyecta también en el terreno del derecho administrativo sancionador, y que exige para su aplicación en este tipo de casos que se den dos requisitos: 1) que el mal causado (transgresión de la norma sobre receta) no sea mayor que el que se trate de evitar (preservación de la salud de la paciente); 2) y que la situación de necesidad no haya sido provocada intencionadamente por el afectado (aquí el conflicto surge por un problema administrativo o informático ajeno a la voluntad de la paciente).

La recomendación legal para este tipo de supuestos es siempre dejar constancia escrita en la farmacia del conjunto de hechos y factores que motivaron la dispensación excepcional y, por supuesto, advertir a la paciente de que en cuanto le sea posible presente la receta en la farmacia. Para lo primero, un instrumento idóneo sería utilizar el Libro de Incidencias de SEFAC (LIFAC).

Finalmente, debe resaltarse que, si se permitiera a la farmacia comunitaria el acceso a la historia clínica del paciente, al menos a la parte farmacoterapéutica, se podría dar, desde luego, mejor solución a conflictos como el puesto de manifiesto en este caso clínico. A este respecto, no debe olvidarse que, conforme prescribe la Ley 44/2003, de 21 de noviembre, de ordenación de las profesiones sanitarias, se debe trabajar en equipo, que es la unidad básica asistencial, y dentro del mismo debe contarse necesariamente con el farmacéutico comunitario.

\section{Referencias bibliográficas}

Comisión de Bioética de SEFAC. Manual de consultas bioéticolegales en la Farmacia Comunitaria, SEFAC, 2012. ISBN 978-84-616-0659-7.

Baixauli V, Abellán F. Cuestiones de conciencia en la farmacia comunitaria, con especial referencia a la dispensación excepcional. En: Libertad de conciencia y medicamento. Una guía práctica, Comares, Granada 2011. ISBN 978-84-9836-792-8.

Gutiérrez Ríos P, Abellán García Sánchez F, Molina Porlán P, Gastelurrutia Garralda MA, Faus Dáder MJ, Martínez Martínez F. Evaluación de la utilidad de un Libro de Incidencias en la farmacia comunitaria (Proyecto LIFAC). Farmacéuticos Comunitarios, 2011, Sep 30;3(3): 127-135. Disponible en: https://www.farmaceuticoscomunitarios.org/es/journal-article/evaluacion-utilidad-libro-incidencias-farmacia-comunitaria-proyecto-lifac 